\title{
Effect of exercise alone or combined with dietary supplements on anthropometric and physical performance measures in community-dwelling elderly people with sarcopenic obesity: A meta-analysis of randomized controlled trials(Review)
}

- Hita-Contreras, F.

- Bueno-Notivol, J.

- Martínez-Amat, A. ${ }^{a}$,

- Cruz-Díaz, D. ${ }^{\mathrm{a}}$,

- Hernandez, A.V.,

- Pérez-López, F.R. ${ }^{\mathrm{e}}$ Email Author

- View Correspondence (jump link)

- ${ }^{a}$ Department of Health Sciences, Faculty of Health Sciences, University of Jaén, Jaén, Spain

- ${ }^{b}$ Department of Psychiatry, Miguel Servet University Hospital, Zaragoza, Spain

- 'University of Connecticut/Hartford Hospital Evidence-Based Practice Center, Hartford, CT 06102, United States

$\underline{\text { View additional affiliations }}$

\section{Author keywords}

Dietary supplementElderlyExerciseMeta-analysisObesityPhysical performanceSarcopeniaSarcopenic obesity

\section{Indexed keywords}

agedappendicular skeletonbody compositiondietary

EMTREE supplementexercisefat massgaitgeriatric patientgrip strengthhumanmeta medical analysismuscle massphysical performancerandomized controlled trial terms: (topic)Reviewsarcopenic obesityskeletal musclesystematic reviewtrunkwaist circumference

- ISSN: 03785122

- CODEN: MATUD

- Source Type: Journal

- Original language: English

- DOI: $10.1016 /$ j.maturitas.2018.07.007

- Document Type: Review

- Publisher: Elsevier Ireland Ltd 